\title{
A case of extragenital linear lichen sclerosus along the lines of Blaschko responding to methotrexate
}

\author{
Mustafa Ürün ${ }^{1 凶}$, Yıldız Gürsel Ürün', Sezgi Sarıkaya Solak ${ }^{1}$ \\ ${ }^{1}$ Department of Dermatology and Venereology, Faculty of Medicine, Trakya University, Edirne, Turkey.
}

\begin{abstract}
Lichen sclerosus, a rare, chronic, inflammatory, mucocutaneous disorder of the genital and extragenital skin, is usually asymptomatic and affects both sexes. The exact cause of lichen sclerosus is unknown. Extragenital lichen sclerosus may be localized or disseminated. Linear extragenital lichen sclerosus following the lines of Blaschko is an exceptionally rare form. A 66-year-old female patient presented with a sclerotic plaque extending from the dorsum of the right hand toward the elbow. The lesion first appeared on the right hand but spread toward the elbow within 1 year. The histological findings were consistent with a diagnosis of lichen sclerosus. We therefore diagnosed extragenital linear lichen sclerosus and achieved a good response with methotrexate.
\end{abstract}

Keywords: lichen sclerosus, lines of Blaschko, methotrexate

Received: 21 January 2020 | Returned for modification: 23 March 2020 | Accepted: 12 April 2020

\section{Introduction}

Lichen sclerosus (LS) is a chronic inflammatory dermatosis of the skin and mucosal surfaces that typically affects the anogenital area, but it may also present with extragenital manifestations (1). Classic extragenital sites include the neck, upper trunk area, and shoulders (2). Extragenital linear lesions are uncommon, especially those in a Blaschkoid distribution.

We present here a rare case of linear extragenital LS that followed the lines of Blaschko from the dorsal aspect of the right middle finger toward the elbow, and that responded to treatment with methotrexate.

\section{Case report}

A 66-year-old female patient presented with a sclerotic plaque extending from the dorsum of the right hand toward the elbow. The lesion first appeared on the right hand but spread toward the elbow within 1 year. A dermatological examination revealed an atrophic and sclerotic plaque that extended linearly from the dorsal aspect of the right middle finger toward the elbow. The segment on the dorsal aspect of the right hand was ivory-colored, and the segment on the arm was light brown (Fig. 1). The oral and genital mucosa were normal. The patient had no personal history or family history of any autoimmune disorders.

Blood parameters (complete hematological study, liver, renal, and thyroid function tests, lipid profile, hepatitis B and C serology, anti-Borrelia antibodies, and antinuclear antibody level) were within normal range, and X-ray imaging showed no pathologies.

The patient was preliminarily diagnosed with morphea and LS, and two biopsy samples were collected from the arm and the dorsum of the right hand. Similar results were obtained following histological examination of the biopsy samples. The histopathology showed thinning of the epidermis, flattening of the rete ridges, hyalinization of the papillary dermis, and inflammatory infiltrate in the papillary dermis-reticular dermis interface (Fig. 2). Based on both clinical and histopathological findings, the pa- tient was diagnosed with extragenital linear LS that followed the lines of Blaschko.

The patient was prescribed topical clobetasol propionate (0.05\%) ointment to use once daily for 2 months, but the lesion failed to respond to this treatment. We then started the patient on $30 \mathrm{mg}$ daily of acitretin. The patient's lesion did not exhibit any changes after 3 months, and flexion contracture occurred in the middle finger. It was then decided to start the patient on methotrexate, which has been successfully used in the treatment of morphea. The initial dose of $5 \mathrm{mg}$ was later increased to $15 \mathrm{mg} /$ week. The lesion started softening after 1 month, and an acceptable response was obtained after 6 months (Fig. 3). There was also a significant improvement in the flexion contracture.

\section{Discussion}

LS is a rare chronic inflammatory disease commonly occurring in the genital region (3). It affects both sexes but is more common in females. Although the etiology of LS has yet to be explained, autoimmune mechanisms, genetic susceptibility, trauma and chronic irritation, infections with Epstein-Barr virus and Borrelia burgdorferi, and hormonal influences have been suggested. Only $6 \%$ of LS cases involve isolated extragenital lesions (1). Extragenital sites may include upper areas of the trunk, neck, shoulders, palms, soles, scalp, and face. These lesions present as erosions, papules, porcelain-white plaques, and wide degrees of sclerosis. Although extragenital LS is mainly asymptomatic, it can occasionally be pruritic (2).

The first to describe a linear form of extragenital LS in 1995 were Izumi and Tajima (4). A small number of cases of linear LS have been reported since then. Among these, patterns corresponding to the lines of Blaschko were noted to have developed. Most skin lesions follow the linear pattern that was first described by Blaschko in 1901. Theselines, referred to as the lines of Blaschko or Blaschko's lines, are a classic example of cutaneous mosaicism. Lichen striatus, lichen planus, lichenoid drug eruption, lupus erythematosus, psoriasis, and morphea are some of the diseases that follow the 


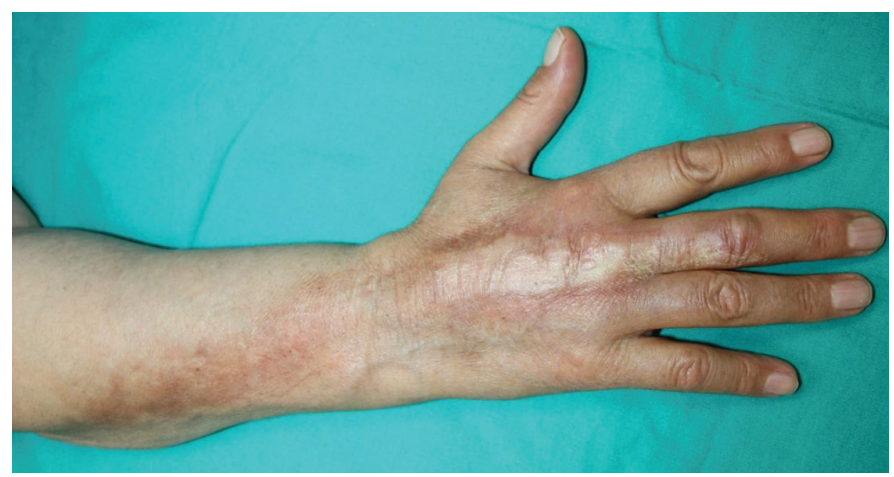

Figure 1 | A sclerotic plaque extending from the dorsum of the right hand toward the elbow.
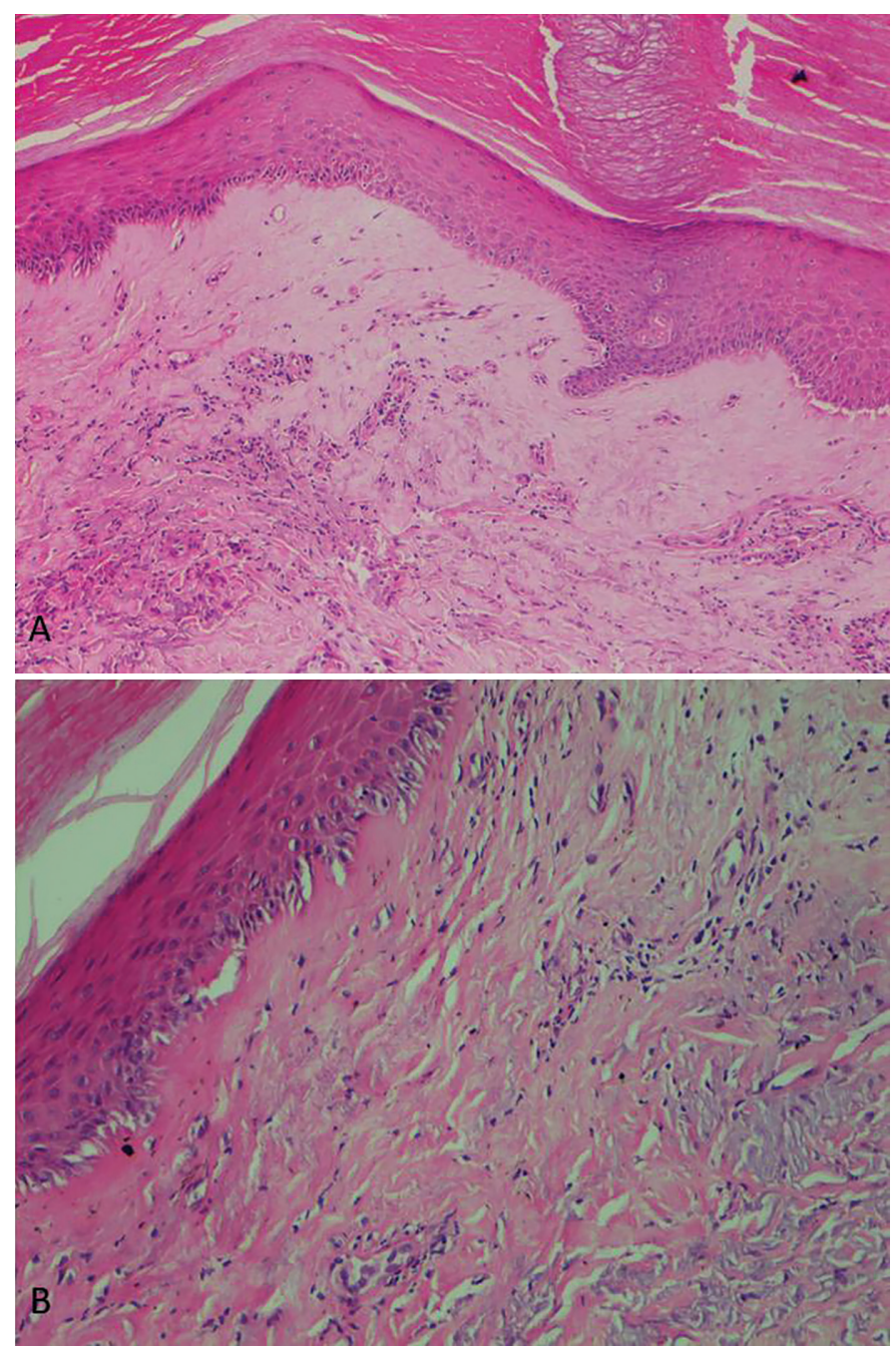

Figure 2 | a) Skin biopsy specimen taken from the dorsum of the right hand (H\&E $\times 100)$ and $b$ ) from the arm $(H \& E \times 200)$. Thinning of the epidermis, flattening of the rete ridges, hyalinization of the papillary dermis, and inflammatory infiltrate in the papillary dermis-reticular dermis interface.

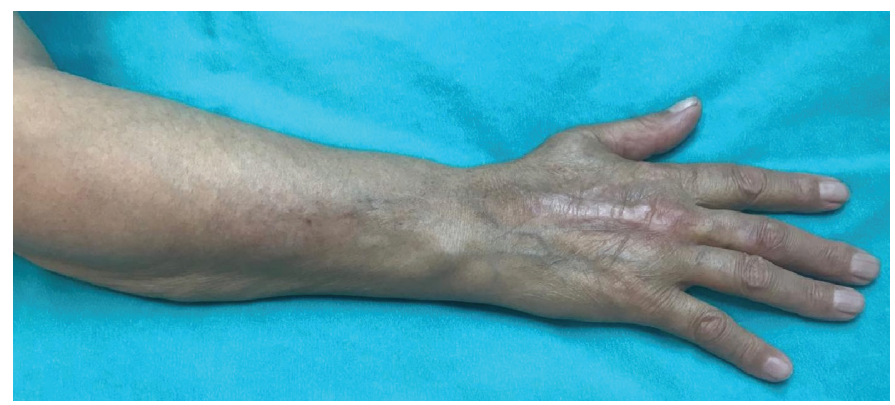

Figure 3 | After 6 months of methotrexate treatment. lines of Blaschko (5). Linear extragenital LS that follows the lines of Blaschko is an exceptionally rare form. Six cases of linear LS that occurred on the trunk, limbs, and face were summarized by Kim and Lee. These followed the lines of Blaschko (6). Extragenital LS's differential diagnosis includes morphea and atrophic lichen planus (ALP) (3). ALP is one of the rarest clinical variants of lichen planus (7). The lower extremities, axilla, glans penis, and trunk are the main sites of involvement (8). A linear form that follows the lines of Blaschko has also been reported (9). Typical lichen planus findings, such as hyperkeratosis, band-like lymphocytic infiltrate in the papillary dermis, and colloid bodies in the dermoepidermal junction, are observed on histopathological examination (7); thus, ALP can be easily discerned from LS. LS has a distinctive histologic pattern. This includes hyperkeratosis and epidermal atrophy with flattening of the rete ridges. Also present are vacuolar interface changes, the loss of elastic fibers, and hyalinization of the lamina propria with an underlying lymphocytic infiltrate (1). Morphea is a chronic disease characterized by well-defined shiny indurated plaque with violaceous borders and skin induration. It is considered that autoimmunity plays a role in the etiology of this disease. Thick homogenized collagen bundles extending into the subcutaneous tissue and inflammation in the reticular dermis are the main histopathological characteristics of morphea (10). The relationship between LS and morphea remains controversial, although LS and morphea have been shown to coexist in some cases. Some authors have asserted that their histopathology and clinical picture are sufficient to distinguish between the two diseases (2). Whereas edema and elastic tissue loss are dominant in LS, changes in the reticular dermis due to inflammation and fibrosis are more prevalent in morphea (11).

Today, there is no definitive treatment for LS (11). Treatment options for extragenital LS include topical corticosteroids, shave excision, low-dose ultraviolet (UV) A1 phototherapy, narrow-band UVB phototherapy, carbon dioxide laser, methotrexate, and acitretin. However, responses to these treatments have been variable $(3,11,12,13)$. Although topical corticosteroids are generally preferred for the initial treatment of LS, extragenital LS does not respond well to topical corticosteroids (12). The administration of topical clobetasol propionate (0.05\%) ointment to our patient for 2 months failed to elicit a response. Our clinic could not provide carbon dioxide laser and local UVA1 phototherapy. Furthermore, shave excision was not suitable because the lesion covered a large area. The patient was therefore started on acitretin. Although there is no consensus on the use of acitretin for extragenital LS in the literature, it has been shown to be successful in some cases $(3,13)$. Our patient was treated with $30 \mathrm{mg}$ of acitretin daily for 3 months. However, in contrast to the findings of previous studies, the lesion did not exhibit any improvement, and flexion contracture occurred in the middle finger. Because flexion contracture developed and the desired response could not be obtained with acitretin, the patient was started on methotrexate, which has been used successfully to treat morphea. The initial dose was 5 $\mathrm{mg}$, which was later increased to $15 \mathrm{mg} /$ week. The lesion started softening after 1 month, and an acceptable response was obtained after 6 months. There was also a significant improvement in flexion contracture. No complications were observed during the treatment process. Low-dose methotrexate in combination with pulsed high-dose corticosteroids is mostly preferred in patients with severe and treatment-resistant generalized extragenital LS (14). Notwithstanding this, the literature describes patients with extragenital LS that were only treated with methotrexate (12). 
In recent years, methotrexate has been used as a new treatment option for sclerotic skin diseases such as scleroderma, morphea, and LS. However, its mechanism of action in these diseases is not completely understood (14). Increased levels of interleukins 2, 4, 6, and 8 and the inhibition of such cytokines are among the possible mechanisms of action of methotrexate in localized scleroderma, which is one of the sclerotic diseases (15). A recent study suggested that low-dose methotrexate's primary immunosuppressive and anti-inflammatory mechanism of action is associated with suppression of the JAK/STAT pathway (16).

\section{References}

1. Fistarol SK, Itin PH. Diagnosis and treatment of lichen sclerosus: an update. Am J Clin Dermatol. 2013;14:27-47.

2. Kim CR, Jung KD, Kim H, Jung M, Byun JY, Lee DY, et al. Linear lichen sclerosus along the Blaschko's line of the face. Ann Dermatol. 2011;23:222-4.

3. Basak PY, Basak K. Lichen sclerosus et atrophicus of the scalp: satisfactory response to acitretin. J Eur Acad Dermatol Venereol. 2002;16:183-5.

4. Izumi T, Tajima S. A case of linear type of lichen sclerosus et atrophicus? J Dermatol. 1995;22:279-82.

5. Molho-Pessach V, Schaffer JV. Blaschko lines and other patterns of cutaneous mosaicism. Clin Dermatol. 2011;29:205-25.

6. Kim YJ, Lee ES. Case of sequentially occurring lesions of facial lichen sclerosus following the lines of Blaschko. J Dermatol. 2007;34:201-4.

7. Krispinsky AJ, Ulman CA. Atrophic lichen planus. J Clin Aesthet Dermatol. 2019; 12:14.

8. Weston G, Payette M. Update on lichen planus and its clinical variants. Int J Womens Dermatol. 2015;1:140-9.

9. Lakshmi C, Divakaran J, Sivaraman A, Srinivas CR. Painful linear atrophic lichen planus along lines of Blaschko. Indian J Dermatol. 2006;51:42-3.

10. Arif T, Adil M, Amin SS, Mahtab A. Concomitant morphea and lichen sclerosus et atrophicus in the same plaque at the site of intramuscular drug injection: an interesting case presentation. Acta Dermatovenerol Alp Pannonica Adriat. 2018; 27:111-3.

\section{Conclusions}

We report a rare case of linear extragenital LS that followed the lines of Blaschko and responded well to treatment with methotrexate. Nonetheless, due to the potential side effects of the drug and based on our experience with this patient, we recommend using methotrexate mainly for patients with treatment-resistant extragenital LS.

11. Diwan NG, Nair AP. Extragenital lichen sclerosus et atrophicus along the lines of Blaschko. Indian Dermatol Online J. 2015;6:342-4.

12. Nayeemuddin F, Yates VM. Lichen sclerosus et atrophicus responding to methotrexate. Clin Exp Dermatol. 2008;3:651-2.

13. Formiga Ade A, Torres Ide S, Rocha Bde O, Silva AO, Follador I, Rêgo VR, et al. Disseminated extragenital lichen sclerosus et atrophicus treated with acitretin. Skinmed. 2014;12:62-3.

14. Kreuter A, Tigges C, Gaifullina R, Kirschke J, Altmeyer P, Gambichler T, et al. Pulsed high-dose corticosteroids combined with low-dose methotrexate treatment in patients with refractory generalized extragenital lichen sclerosus. Arch Dermatol. 2009;145:1303-8.

15. Kreuter A, Gambichler T, Breuckmann F, Rotterdam S, Freitag M, Stuecker M, et al. Pulsed high-dose corticosteroids combined with low-dose methotrexate in severe localized scleroderma. Arch Dermatol. 2005;141: 847-52.

16. Thomas S, Fisher KH, Snowden JA, Danson SJ, Brown S, Zeidler MP. Methotrexate is a JAK/STAT pathway inhibitor. PLoS One. 2015;10:e0130078. 